\title{
Numerical modeling for heatsink emissions in power electronics
}

\author{
J. Kulanayagam ${ }^{1}$, J. H. Hagmann ${ }^{1}$, S. Schenke ${ }^{1}$, K. F. Hoffmann ${ }^{2}$, and S. Dickmann ${ }^{1}$ \\ ${ }^{1}$ Institute for Fundamentals of Electrical Engineering, Germany \\ ${ }^{2}$ Institute for Power Electronics Helmut-Schmidt-University/University of the Federal Armed Forces Hamburg, Germany
}

Correspondence to: J. Kulanayagam (kulanayagam@hsu-hamburg.de)

\begin{abstract}
The parasitic coupling between power semiconductors and the heat sink is responsible for noise current in Switching Mode Power Supply (SMPS) systems. In this paper, the variations in the radiation characteristics of heatsinks are investigated with respect to their geometries by use of numerical models. Analyses are facilitated by using a mopole antenna as an EMI receiver and by using simplified heatsink models as EMI transmitters to model the heatsink radiated emissions. In addition, the analysis is confirmed using laboratory measurements.
\end{abstract}

\section{Introduction}

Power-switching semiconductors (MOSFET's/IGBT's) are usually mounted on heat sinks to keep the temperature below an acceptable limit. Parasitic capacitances can be created between the switching devices and the heatsink. The electric isolator (mica) acts as a dielectric of these capacitances. The result of this parasitic effects, the common-mode EMI is generated in Switching Mode Power Supply circuits (Tihanyi, 1995). If this current contains a high number of harmonic components and its flows through the heatsink, the heatsink becomes an antenna. However, the maximum radiation occurs at different frequencies depending on the heatsink geometry (Brench, 1994; Das and Roy, 1998). In consequence, this current could violate the EMC standards due to radiation. Therefore the heat sink should be well designed. A typical heatsink configuration is shown in Fig. 1.

In this paper, the variations in the radiation characteristics of non-grounded and grounded heatsinks are investigated. A previous paper have shown that very little difference in the general electromagnetic behaviors of a finned heatsink and a heatsink as a solid block (Dawson et al., 2001). In consequence, a heatsink as a solid block allows a much simpler simulation model.

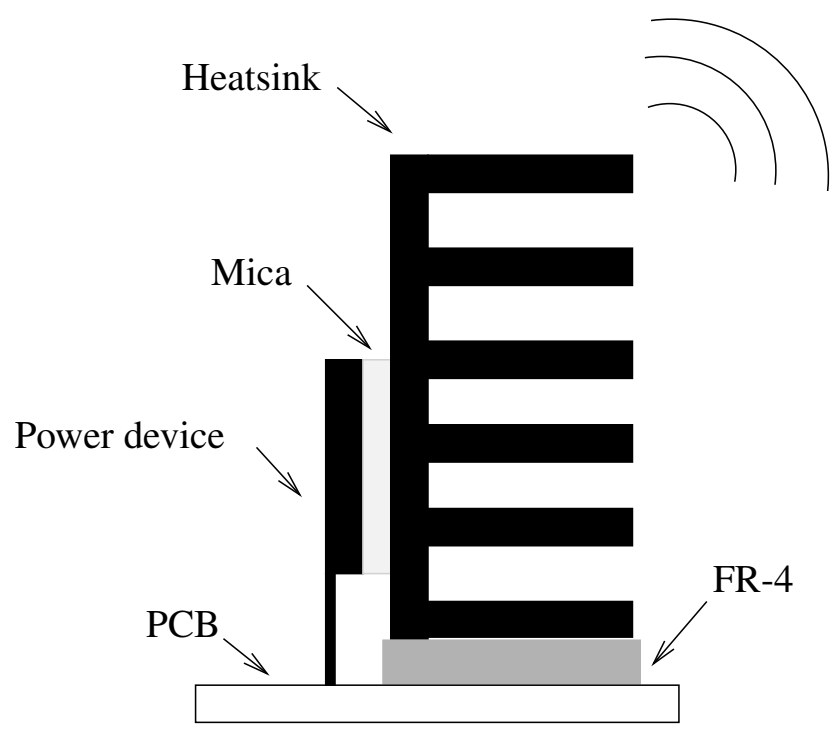

Fig. 1. Schematic representation of parasitic capacitance.

\section{Experimental setup}

In order to represent a ground plane, a rectangular piece of aluminum $(2 \times 1.5 \mathrm{~m})$ was used. The ground plane was placed on a wooden table inside an anechoic chamber. The edges of the ground plane were covered by microwave absorbers to limit the effect of reflections from nearby objects (Dawson et al., 2001). The heatsink block $(88 \times 34 \times 53 \mathrm{~mm})$ was supported by a small dielectric block $88 \times 34 \times 5 \mathrm{~mm}$ (not included in the numerical model) over a ground plane. A MOSFET (TO-247) was placed on the heatsink. The electric isolator was inserted between the case of the power device and the heatsink. The thickness and dielectric constant of Mica are $0.05 \mathrm{~mm}$ and 3.5, respectively. In order to create the excitation source the SMA- type connector was connected to the pin (drain) of the semiconductor. 


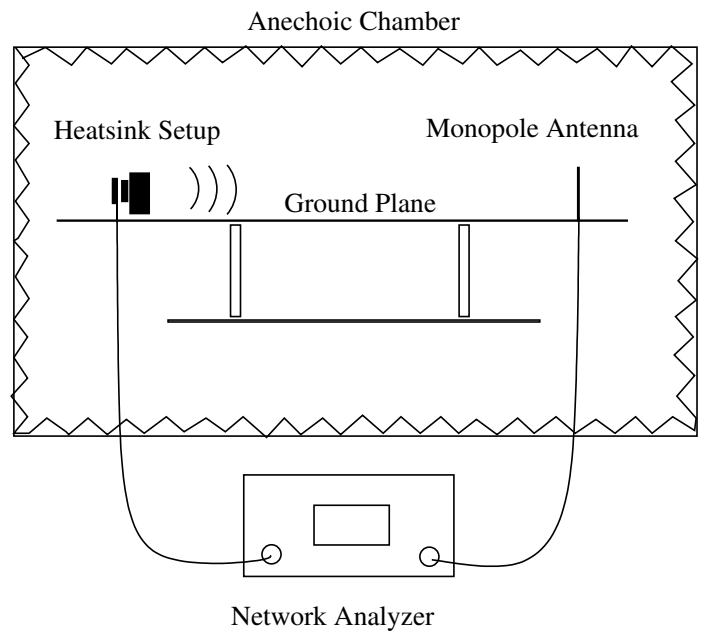

Fig. 2. Experimental setup (heatsink frontally and monopole antenna vertically).

The access of the connector was connected to the network analyzer via coaxial cable. A $7.5 \mathrm{~cm}$ high monopole antenna ( $1 \mathrm{~mm}$ diameter), $1 \mathrm{~m}$ from the centre of the heatsink block, sensed the radiated emissions from the heatsink for model validation purposes. The network analyzer was used to determine the transmission parameters from the heatsink to the monopole antenna $\left(S_{21}\right)$ in order to validate the model. The simplified model (without microwave absorbers around the table) shown in Fig. 2 is used for experimental purposes.

\section{Numerical modeling}

In order to compute the electromagnetic field outside the heatsink model in the far field environment, CST Microwave Studio was used. In the numerical model all metallic parts are considered as Perfect Electric Conductor (PEC) materials. The heatsink block was placed over the infinite ground plane without the small dielectric support. It was assumed that the permittivity of the materials remained constant in the considered frequency range.

\section{Results and discussions}

Non-grounded and grounded heatsink models are necessary to analyze the differing process conditions of a heatsink.

\subsection{Non-grounded heatsink}

The noise current is generated due to the parasitic capacitances of the heatsink design. This noise current can be calculated according to the following assumption. In the first approach for low frequencies, the capacitance between the case of the power device and the heatsink $C_{\mathrm{dh}}$ is much greater than the capacitance between the heatsink and ground plane
Non-grounded heatsink

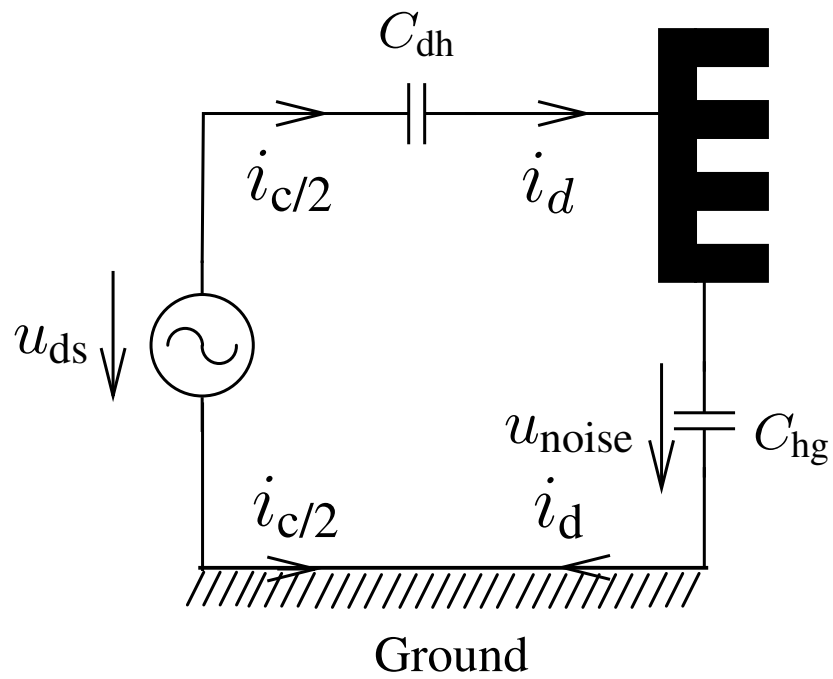

Fig. 3. Noise currents in the non-grounded heatsink.

$C_{\text {hg }}$. The common-mode current is not taken into account in this case. Firstly, the simplified non-grounded heatsink model (see Fig. 3) is considered.

The differential-mode current can be calculated:

$i_{\mathrm{d}}(t)=C_{\mathrm{hg}} \cdot \frac{\mathrm{d} u_{\text {noise }}}{\mathrm{d} t}$.

For low frequencies $C_{\mathrm{dh}}>>C_{\mathrm{hg}}$, the noise voltage can be calculated as follows

$u_{\text {noise }} \simeq u_{\mathrm{ds}}$

The differential-mode current is given by following equation

$i_{\mathrm{d}}(t) \simeq C_{\mathrm{hg}} \cdot \frac{\mathrm{d} u_{\mathrm{ds}}}{\mathrm{d} t}$.

Four configurations were made for the non-grounded heatsink block model. Firstly, the heatsink block was supported by a small dielectric block with the relative permittivity $\varepsilon_{r}=1$ over a ground plane. A MOSFET was placed on the heatsink. The electric isolator (Mica) was inserted between the case of the MOSFET and the heatsink. Fig. 4 shows the $S_{21}$ parameter for the first configuration of the measured and simulated coupling $\left(S_{21}\right)$ for the frontally and laterally polarized heatsink. In order to compare the radiated emissions of the heatsink, the measured result of the frontally polarized heatsink was used as the reference curve for the non-grounded and grounded heatsink.

In order to change the parasitic capacitance between the heatsink and the power device, the thickness of the Mica was doubled in the second configuration. The results of the second configuration are shown in Fig. 5. As expected, the results of the first and second configuration are the same. The 


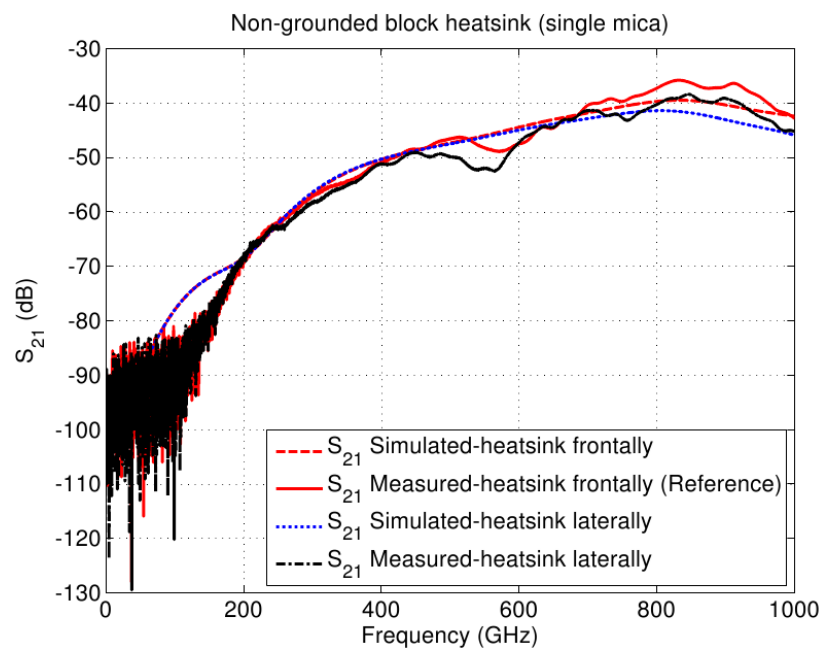

Fig. 4. Comparison of measured and simulated results of $S_{21}$ for the frontally and laterally polarized non-grounded heatsink with single Mica.

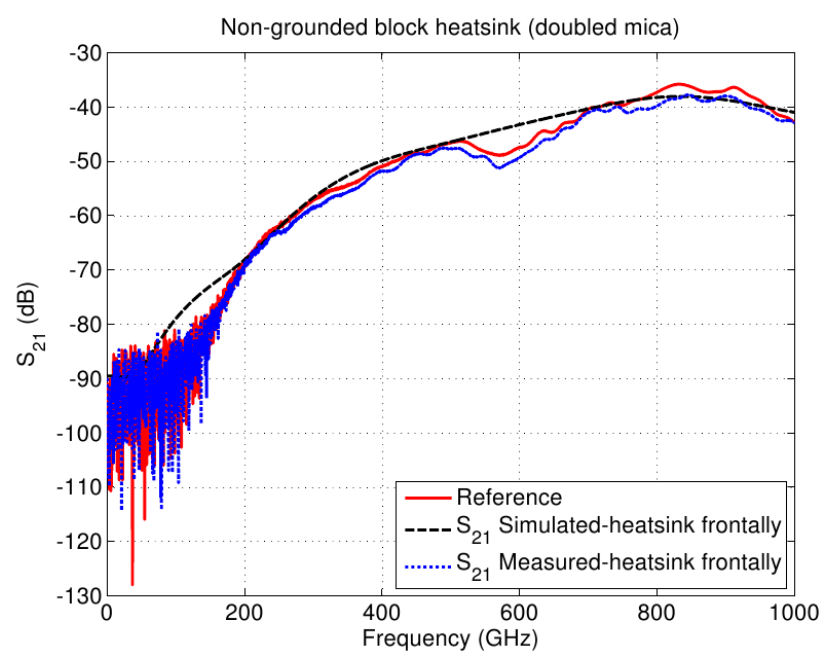

Fig. 5. Comparison of measured and simulated results of $S_{21}$ for the frontally polarized non-grounded heatsink with two Micas.

Fig. 4 and the Fig. 5 show nearly $8 \mathrm{~dB}$ deviation in the frequency range 500-580 $\mathrm{MHz}$ between measurement and simulation. In a real situation, there are always some reflections due to objects inside the anechoic chamber.

The difference between the first and the third configuration is the heatsink block was supported by a Polyvinyl Chloride (PVC) block over the ground to change the capacitance between the heatsink and ground plane. The measured and simulated results of the third configuration are shown in Fig. 6. The Fig. 6 shows nearly $5 \mathrm{~dB}$ deviation in the frequency range 800-900 $\mathrm{MHz}$ between measurement and simulation. The radiated emission was reduced in the frequency range due to the reduction of the impedance between the heatsink and ground plane. In consequence, the radiated emissions were now less than the reference model.

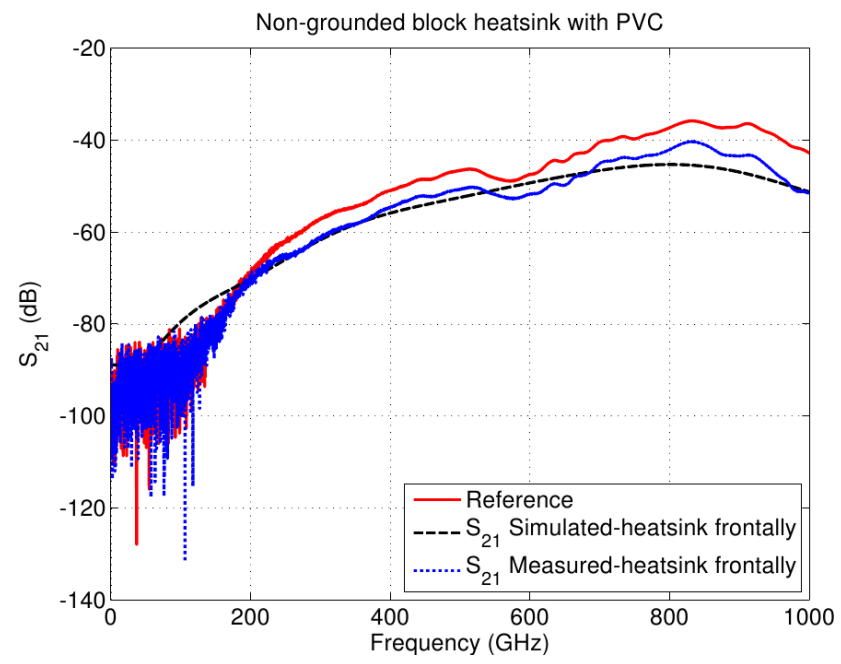

Fig. 6. Comparison of measured and simulated results of $S_{21}$ for the frontally polarized non-grounded heatsink supported by PVC.

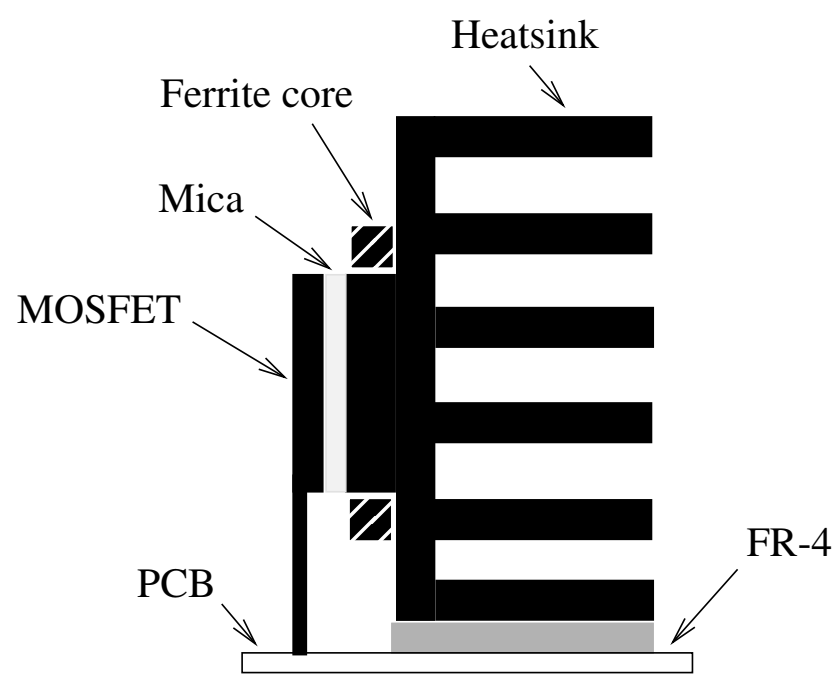

Fig. 7. Schematic representation of the filter design.

Furthermore, by adding a ferrite core around the metallic cylinder of the heatsink (see Fig. 7) the input impedance of the heatsink can be increased (Kulanayagam et al., 2011). In consequence, the noise current can be minimized, which results in reduced radiation from the heatsink.

In the fourth configuration two different ferrite cores were used, which consisted of different material characteristics (see Fig. 8). The radiated emissions were reduced by adding the ferrite core around the current path between the MOSFET and the heatsink. 


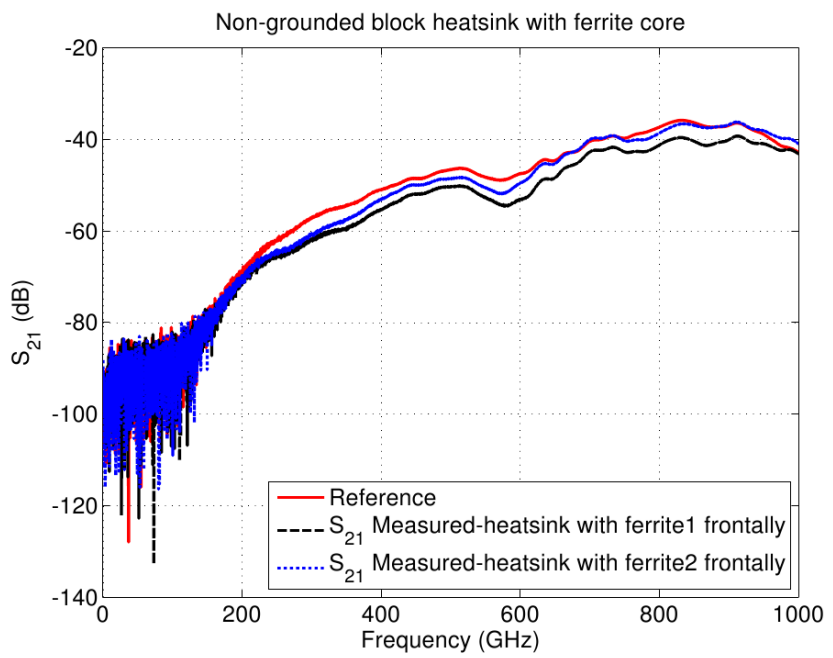

Fig. 8. Measured results of $S_{21}$ for the frontally polarized nongrounded heatsink for two different ferrite cores.

\section{Grounded heatsink}

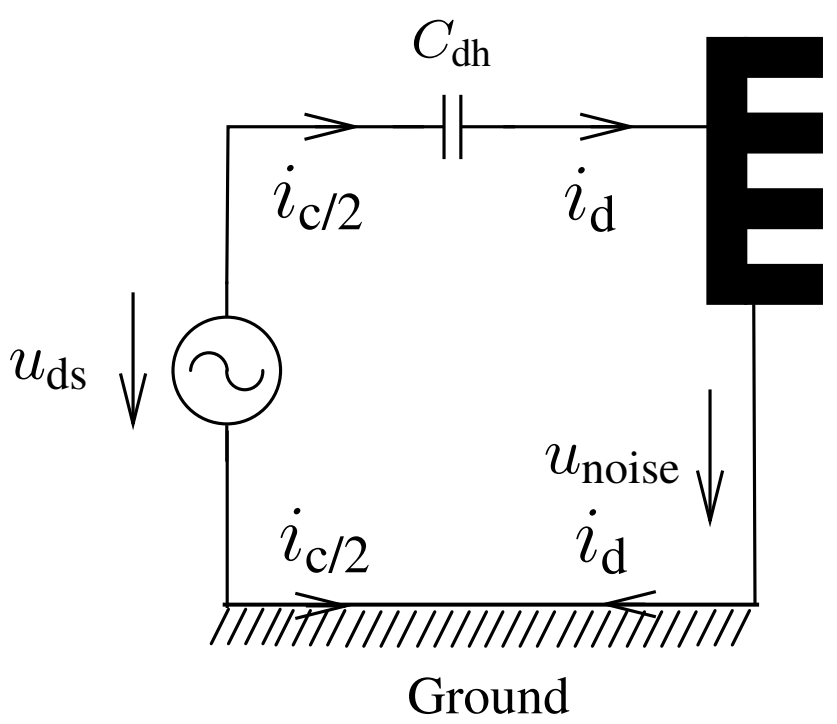

Fig. 9. Noise currents in the grounded heatsink.

\subsection{Grounded heatsink}

Finally, the simplified grounded heatsink model (see Fig. 9) is considered. The differential-mode current is given by

$i_{\mathrm{d}}(t)=C_{\mathrm{dh}} \cdot \frac{\mathrm{d} u_{\mathrm{ds}}}{\mathrm{d} t}$.

The noise voltage can be calculated as follows

$u_{\text {noise }} \simeq 0$.

Fig. 10 compares the simulated and measured $S_{21}$ when the heatsink is grounded at both sides of the heatsink with copper tabs $(8 \times 1 \times 5 \mathrm{~mm})$. At frequencies below $600 \mathrm{MHz}$, the

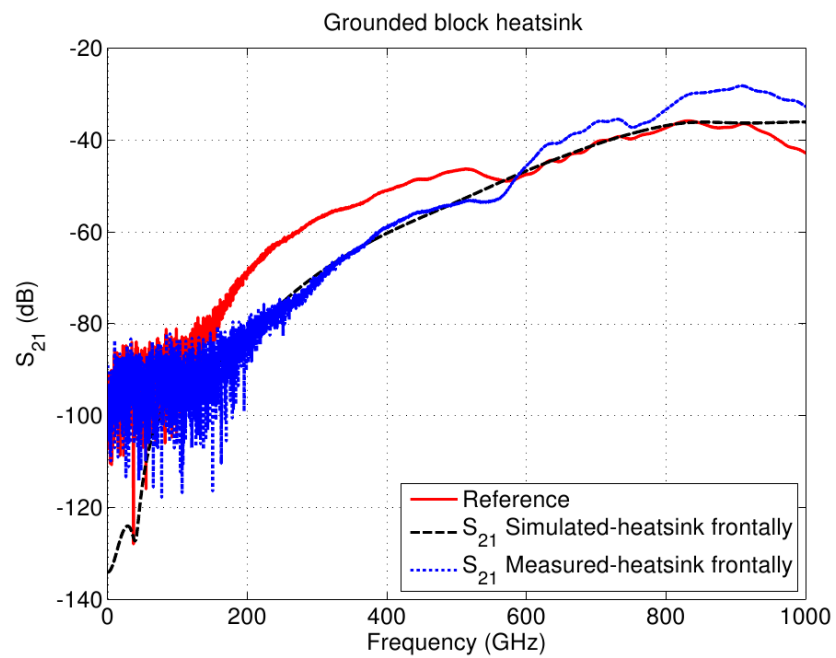

Fig. 10. Comparison of measured and simulated results of $S_{21}$ for the frontally polarized grounded heatsink with a single Mica.

grounded heatsink is effective. The grounding wires have a high impedance in the high frequency range. In consequence, above frequencies of $600 \mathrm{MHz}$, the radiated emissions are higher than with the reference setup. The choice of the grounding wire is very important in SMPS circuits. It has also been observed that the measured noise floor is higher up to a frequency of $200 \mathrm{MHz}$ for the non-grounded and grounded heatsink design.

\section{Conclusions}

The main objective of this work was to investigate the radiated EMI of the heatsink. The work is based on numerical modeling and the experimental EMI measurements for the non-grounded and the grounded heatsink.

The results of the numerical modeling and the experimental EMI measurements were compared for the non-grounded heatsink with a small dielectric support, varying thickness of the dielectric material layer between the power device and the non-grounded heatsink, the non-grounded heatsink with PVC support and the grounded heatsink with a small dielectric support. The results of the experimental EMI measurements were also presented for the adding of the ferrite core between the power device and the non-grounded heatsink. The capacitance between the heatsink and the ground plane is responsible for the EMI radiation of the non-grounded heatsink, while the capacitance between the heatsink and power semiconductor is responsible for the grounded heatsink. By adding the ferrite core between the extended heatsink and the power device, the radiated emissions from the non-grounded heatsink can be minimized. In order to reduce the radiated emissions, the heatsink should be grounded. The radiated emissions from the grounded heatsink depend on the grounding wires, which 
are connected from the heatsink to the ground plane. The grounding wire should have minimal impedance, otherwise it can become an efficient antenna. A good correlation between the numerical model and measurements was obtained.

\section{References}

Brench, C.: Heatsink radiation as a function of geometry, in: IEEE International Symposium on Electromagnetic Compatibility, 105-109, 1994.

Das, S. and Roy, T.: An investigation on radiated emissions from heatsinks, in: IEEE International Symposium on Electromagnetic Compatibility, 2, 784-789, 1998.
Dawson, J., Marvin, A., Porter, S., Nothofer, A., Will, J., and Hopkins, S.: The effect of grounding on radiated emissions from heatsinks, in: IEEE International Symposium on Electromagnetic Compatibility, 2, 1248-1252, 2001.

Kulanayagam, J., Hagmann, J. H., Hoffmann, K. F., and Dickmann, S.: Reduction of heat sink common-mode currents in switching mode power supply circuits, Adv. Radio Sci., 9, 317-321, doi:10.5194/ars-9-317-2011, 2011.

Tihanyi, L.: Electromagnetic Compatibility in Power Electronics, J. K. Eckert \& Company, Inc., Sarasota, Florida, 1995. 